\title{
Elemente und Ephemeride des Planeten (113) Amalthea.
}

Von W. Luther.

Durch Hinzuftigung der Störungen durch Jupiter und Saturn zu den Elementen, mit denen auch die Oppositionsephemeride in A. N. 3062 berechnet war, erhielt ich die folgenden Elemente und Ephemeride der Amalthea für die bevorstehende Opposition.

Epoche 1893 Marz 2 1.0 M. Z. Berlin.

$$
\begin{aligned}
& M=342^{\circ} 55^{\prime} \quad 9: 9
\end{aligned}
$$

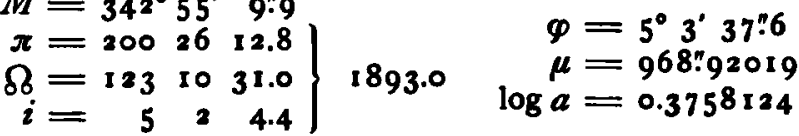

Ephemeride für I $2^{\text {h }}$ M. Z. Berlin.

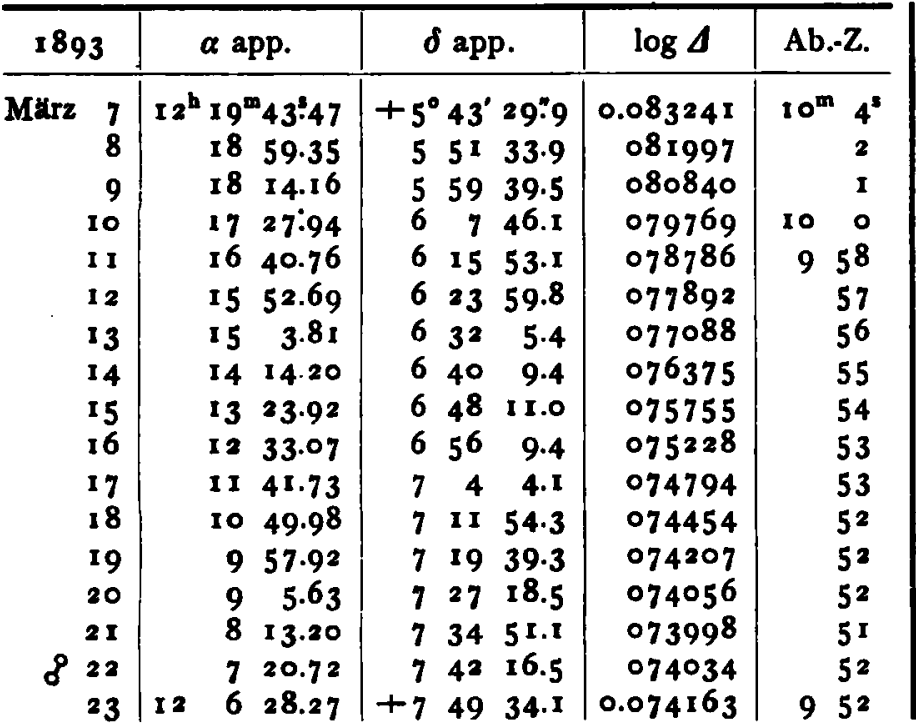

\begin{tabular}{|c|c|c|c|c|c|c|}
\hline \multirow{2}{*}{$\frac{1893}{\text { Mărz } 23}$} & \multicolumn{2}{|c|}{$\alpha$ app. } & \multicolumn{2}{|c|}{$\delta$ app. } & $\log \Delta$ & Ab.-Z. \\
\hline & $12^{h}$ & $6^{m} 8: 27$ & $+7^{\circ} 49$ & $34: 1$ & $0.074 \cdot 163$ & $9^{m} 2^{s}$ \\
\hline 2 & & $5 \quad 35.95$ & $75^{6}$ & 43.3 & $0743^{8} 5$ & $5^{2}$ \\
\hline 2 & & 443.82 & 83 & 43.6 & & $5^{2}$ \\
\hline & & 351.99 & 810 & $34 \cdot 3$ & 075104 & 53 \\
\hline 2 & & $30.5^{2}$ & $8 \div 7$ & 15.0 & 07 & 54 \\
\hline 2 & & 29.50 & 823 & 45.2 & 076 & 54 \\
\hline 2 & & I 19.00 & 830 & 4.4 & 076857 & 55 \\
\hline 3 & 12 & 029.10 & $83^{6}$ & 12.3 & 0776 & 56 \\
\hline 3 & 115 & 5939.87 & 842 & 8.3 & 07 & $5^{8}$ \\
\hline pril & & $\begin{array}{lll}58 & 51.3^{8}\end{array}$ & 847 & 52.2 & 079392 & 959 \\
\hline & & $5^{8} \quad 3.70$ & 853 & 23.5 & 080405 & Io 0 \\
\hline & & $57 \times 6.90$ & 858 & 41.9 & $08 \times 500$ & 2 \\
\hline & & $5^{6} 31.05$ & 9 & 47.0 & 082674 & 3 \\
\hline & & 5546.21 & 9 & 38.7 & 083927 & 5 \\
\hline & & $\begin{array}{ll}55 & 2.44\end{array}$ & $9 \quad 13$ & 16.6 & 085257 & 7 \\
\hline & & 54 I 9.8 I & 917 & 40.4 & 08 & \\
\hline & I I & $533^{8.38}$ & $+92 x$ & 49.9 & $0.088 \times 40$ & IO II \\
\hline
\end{tabular}

Dusseldorf 1892 Dec. 31 .

Grösse $10 \% 4$.

Wilhelm Luther.

Beobachtungen des Cometen 1892 I

angestellt am is Zöller der Pulkowaer Sternwarte von F. Rens.

\begin{tabular}{|c|c|c|c|c|c|c|c|c|c|c|c|c|c|c|c|}
\hline \multicolumn{2}{|c|}{1892} & M. Z. Pulk. & $\Delta a$ & \multicolumn{2}{|c|}{$\Delta \delta$} & Vgl. & $\alpha$ app. & $\log p \cdot \Delta$ & \multicolumn{3}{|c|}{$\delta$ app. } & $\log p \cdot 4$ & \multicolumn{2}{|c|}{ Red. ad 1. app. } & - \\
\hline Ial & & $12^{h} 29^{m} 42^{s}$ & $-I^{m} 20.96$ & $-I^{\prime}$ & 8.8 & 8 & $23^{h} 3^{8^{m}} 3^{2}: 71$ & 34 & +34 & 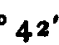 & 5 & 0.835 & $-0: 37$ & $-11: 5$ & 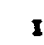 \\
\hline & 13 & $\begin{array}{lll}12 & 53 & 34\end{array}$ & to 55.80 & -0 & 2 t.I & 8 & o 5939.5 I & & $+5^{2}$ & $5 x$ & 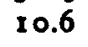 & & & +1.0 & 2 \\
\hline & 15 & 111846 & $+05^{2}$ & -2 & 55.9 & 8 & - 5822.82 & & +52 & 55 & 57.7 & 49 & +2.16 & +1.5 & 2 \\
\hline & $\mathbf{I}$ & $\begin{array}{lll}11 & 24 & 53\end{array}$ & -124.73 & +1 & 57 & 8 & - 56 & & +53 & 0 & & & .26 & +2.3 & $\mathbf{J}$ \\
\hline & 19 & 101921 & +1 & +2 & & 8 & $\circ 5518$ & $9 \mathrm{n}$ & +53 & $\mathbf{I}$ & 46.2 & & $+2.3 x$ & +2.7 & 4 \\
\hline & 2 & $10 \quad 4659$ & $-0 \quad 37.95$ & +3 & 10.4 & 8 & - 5330 & & +53 & 2 & 32.5 & & +2.37 & $+3 \cdot 3$ & 4 \\
\hline & 23 & $10293^{8}$ & to 7.04 & +2 & 1.6 & 8 & - 5135 & 9.6 & +53 & $\mathbf{I}$ & 4 & & +2.44 & +4.0 & 5 \\
\hline & 2 & $10 \quad 4 \quad 57$ & $-05^{2}$ & +1 & 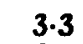 & 8 & - $503^{6}$ & & +53 & $\circ$ & & & 47 & +4.2 & \\
\hline & 2 & 94814 & -15 & -0 & 16. & 8 & - 493 & & +52 & 59 & 28.3 & & +2.50 & +4.5 & \\
\hline & & 95052 & -25 & -2 & 1.2 & 6 & $\begin{array}{lll}0 & 48 & 30.68\end{array}$ & & $+5^{2}$ & 57 & 43 & & +2.53 & +4.8 & \\
\hline & 2 & 9504 & -119.56 & $+I$ & I 5.1 & 8 & - 45 & & +52 & 50 & 4.1 & & +2.62 & +6.0 & 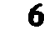 \\
\hline & 30 & 9 II 15 & to 29.34 & +2 & 20 & 8 & $\begin{array}{lll}044 & 0.34\end{array}$ & 9.6 & +52 & 46 & 45.9 & & +2.65 & +6.4 & $y$ \\
\hline 8 & 8 & 91621 & -053 & -0 & $56 . x$ & 8 & - $3^{2} 40.3^{2}$ & & +51 & 57 & & & +2.84 & +9.7 & \\
\hline & Io & $\begin{array}{lll}8 & 41 & 34\end{array}$ & -I 27.95 & +2 & 29.2 & 8 & 030 & & $+5 I$ & 42 & 0.9 & & +2.87 & +10.5 & 9 \\
\hline & I & 92432 & +13 & +3 & 14.1 & 8 & - 2323.75 & & +50 & 55 & 26.5 & & +2.91 & +12.5 & 10 \\
\hline & 16 & 82123 & -116.19 & +3 & 5.1 & 6 & $\begin{array}{lll}0 & 22 & 7.94\end{array}$ & & +50 & 45 & 24.5 & $0.5 \times 8$ & +2.93 & + 12.8 & II \\
\hline
\end{tabular}




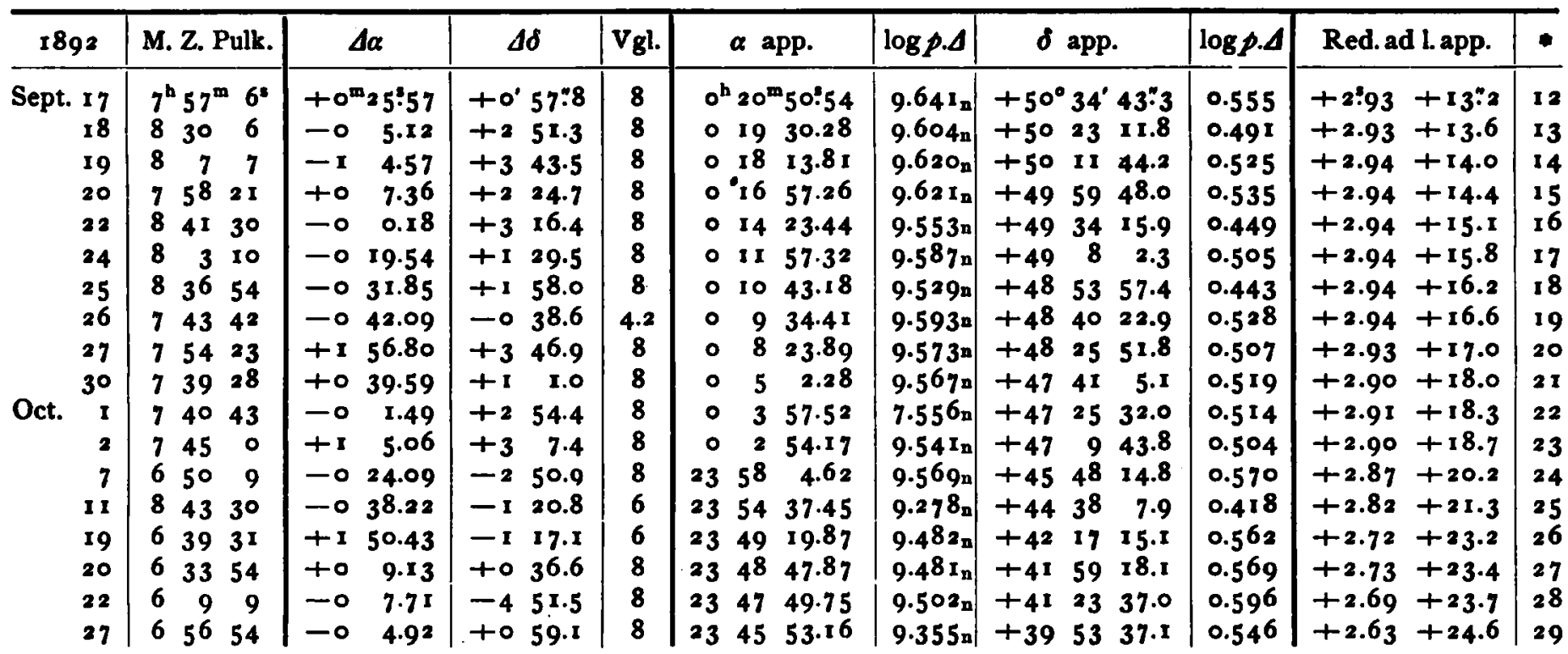

Beobachtet wurde nur Mai 25 bei Feldbeleuchtung, an den ubrigen Abenden an hellen Făden. Vergrösserung 2 ro oder 3 ro, je nach dem Zustande der Bilder.

Die Positionen der Vergleichsterne verdanke ich Herrn Romberg, der dieselben meist gleich oder wenige Tage nach der Cometenbeobachtung am Meridiankreise bestimmte. Die in Klammern eingeschlossenen Cataloge sind bei der Ableitung der mittleren Oerter nicht hinzugezogen.

Mittlere Oerter der Vergleichsterne für 1892.0 .

\begin{tabular}{|c|c|c|c|c|c|c|c|c|}
\hline$*$ & Gr. & Epoche & $\alpha \mathbf{r}$ & 392.0 & $\delta 18$ & 892.0 & Bb. & Sonstige Quellen \\
\hline 1 & $9 \cdot 3$ & 1892.7 & $23^{\mathrm{h}} 3$ & ${ }^{m} 54^{5} \cdot 04$ & $+34^{\circ} 4$ & $44^{\prime} 10^{n ! 8}$ & 2 & \\
\hline 2 & 8.8 & 1892.7 & 0.5 & 41.63 & +525 & 5130.7 & 3 & [AG. Cambr. 50I] \\
\hline 3 & 9.2 & 1892.7 & 05 & 28.27 & +525 & 5852.1 & 3 & \\
\hline 4 & 9.2 & 1892.7 & 05 & 5.89 & +525 & 5918.8 & 3 & \\
\hline 5 & 8.2 & 1892.7 & 05 & 26.25 & +525 & 5940.3 & 3 & [AG. Cambr. 43I] \\
\hline 6 & 9.4 & 1892.7 & 04 & $25.5 \mathrm{I}$ & +524 & $48 \quad 43.0$ & 3 & \\
\hline 7 & 9.1 & 1892.7 & 043 & 28.35 & +524 & 4412.8 & 3 & \\
\hline 8 & 8.7 & 1892.7 & 03 & $3 \mathrm{r.42}$ & $+5=5$ & $58 \quad 13.8$ & 3 & [AOe. 584; AG. Cambr. 277] \\
\hline 9 & 9.1 & 1892.7 & 03 & 29.00 & +533 & 3921.2 & 3 & [AOe. $549 ;$ AG. Cambr. 262 ] \\
\hline 10 & 8.9 & 1892.8 & 021 & 50.27 & +505 & 5159.9 & 3 & [AOe. $366 ;$ AG. Cambr. 178$]$ \\
\hline 11 & 9.1 & 1892.8 & 023 & 21.20 & +504 & $\begin{array}{lll}42 & 6.6\end{array}$ & 3 & [BB. VI +50:82；AG. Cambr. I88] \\
\hline 12 & 9.4 & 1892.8 & 020 & 22.04 & +503 & 3332.3 & 2 & \\
\hline 13 & 9.I & 1892.8 & o Is & 32.47 & +502 & 206.9 & 3 & \\
\hline 14 & 8.4 & 1892.7 & - If & 15.44 & +50 & 746.7 & 3 & [AG. Cambr. 163] \\
\hline 15 & 9.2 & 1892.7 & - It & 46.96 & +495 & 578.9 & 3 & \\
\hline 16 & 7.9 & 1892.8 & - I & 20.68 & +493 & 3044.4 & 3 & [BB. VI +49:50] \\
\hline 17 & 9.0 & 1892.8 & - I 1 & 13.92 & +49 & 617.0 & 2 & \\
\hline 18 & 6.5 & 1892.8 & 0 I & 12.09 & +485 & $\begin{array}{lll}5 \times & 43.2\end{array}$ & 3 & [Lal. 226; Arm 26; Paris 22 I] \\
\hline 19 & 8.7 & 1892.8 & o Ic & 13.56 & +484 & 40. 44.9 & $\mathbf{2}$ & \\
\hline 20 & 9.3 & 1892.8 & - 6 & 24.16 & $+48=$ & 2147.9 & 2 & \\
\hline 21 & 9.3 & 1892.8 & 04 & 19.79 & +473 & 3946.1 & $\mathbf{I}$ & \\
\hline 22 & 8.7 & $\times 892.8$ & 03 & 56.10 & $+47=$ & 2219.3 & 2 & \\
\hline 23 & 8.3 & 1892.8 & & $46.2 \mathrm{I}$ & +47 & $\begin{array}{ll}6 & 17.7\end{array}$ & 3 & [AOe. $264: 2-13$ ] \\
\hline 24 & 9.5 & 1892.8 & 2358 & 25.84 & +455 & $5 \circ 45.5$ & 3 & \\
\hline 25 & 6.0 & 1892.8 & 235 & 12.85 & +443 & 397.4 & 3 & [W, 23.1121; Gro. 4194; Rad. 6255; Rogers \\
\hline 26 & 9.1 & 1892.8 & 234 & 26.72 & +421 & 189.0 & 3 & 1209 ; 10 yr. 4043] \\
\hline 27 & 9.5 & 1892.8 & 2348 & 36.01 & $+4 \times 5$ & 5818.1 & 2 & \\
\hline 28 & 8.8 & 1878.7 & $234 t$ & 54.77 & +412 & $28 \quad 4.8$ & 2 & Romb. $55^{83}$ \\
\hline 29 & 9.5 & 1892.9 & 234 & $55 \cdot 45$ & +395 & $52 \quad 13.4$ & I & \\
\hline
\end{tabular}


Eine Vergleichung der sieben in obigem Verzeichnisse enthaltenen Cambridger Zonensterne mit den Pulkowaer Positionen giebt folgende Unterschiede im Sinne (Romb.-Cambr.) :

$\begin{array}{cccccc}* & \Delta \alpha & \Delta \delta & * & \Delta \alpha & \Delta \delta \\ 2 & -0.10 & +0.5 & 10 & 0.00 & -0.4 \\ 5 & -0.12 & -0.1 & 11 & -0.25 & -0.8 \\ 8 & -0.08 & -0.4 & 14 & -0.21 & -0.7 \\ 9 & -0.22 & -0.4 & \text { Mittel }:-0.14 & -0.3\end{array}$

Vergleichung der Beobachtungen mit der Ephemeride von Berberich (A. N. 3110, 3120, 3128)

(Beob. - Rechn.):

\begin{tabular}{c|c|c|}
\hline \multicolumn{1}{c|}{1892} & $\Delta a$ & $\Delta \delta$ \\
\hline Mai 25 & -0.12 & -3.1 \\
Aug. 13 & -0.71 & -6.0 \\
15 & -0.76 & -6.8 \\
18 & -0.80 & -7.6 \\
19 & -0.75 & -6.2 \\
21 & -0.65 & -6.2 \\
23 & -0.80 & -7.4 \\
24 & -0.78 & -8.9 \\
25 & -0.65 & -8.4 \\
$\left.26^{*}\right)$ & -0.75 & -8.2 \\
29 & -0.79 & -7.7 \\
30 & -0.70 & -8.5
\end{tabular}

\begin{tabular}{r|c|c}
\hline \multicolumn{1}{c|}{1892} & $\Delta \alpha$ & $\Delta \delta$ \\
\hline Sept. 8 & -0.56 & -8.4 \\
10 & -0.54 & -9.9 \\
15 & -0.33 & -10.2 \\
16 & -0.49 & -10.5 \\
17 & -0.41 & -10.2 \\
18 & -0.56 & -10.9 \\
19 & -0.53 & -10.4 \\
20 & -0.49 & -9.6 \\
22 & -0.18 & -10.3 \\
24 & -0.19 & -11.3 \\
25 & -0.14 & $-10: 2$ \\
& &
\end{tabular}

\begin{tabular}{|c|c|c|}
\hline 1892 & $\Delta \alpha$ & $\Delta \delta$ \\
\hline Sept. 26 & -0.25 & - 9.6 \\
\hline & -0.14 & -10.3 \\
\hline & -0.17 & -9.4 \\
\hline Oct. & -0.15 & -10.9 \\
\hline & -0.20 & -10.3 \\
\hline & -0.03 & -12.4 \\
\hline & +0.02 & -10.8 \\
\hline & +0.13 & -9.0 \\
\hline & +0.11 & -9.9 \\
\hline & +0.34 & -9.6 \\
\hline & +0.59 & -9.0 \\
\hline
\end{tabular}

Bemerkungen.

Mai 25. Der Comet ist noch so hell, dass er bei Feldbeleuchtung beobachtet werden kann. In der hellen Dämmerung lässt sich nur eine kleine Coma und ein Kern $\mathrm{rom}^{\mathrm{m}}-10 \mathrm{~m} \cdot 5$ erkennen, der etwas excentrisch im westlichen Theile der Nebelhülle sitzt.

Aug. 13. Der Comet erscheint als heller Nebel mit Kern. - Aug. 15. Eine ausgedehnte Nebelhülle scheint den Kern des Cometen zu umgeben, doch lässt sich die Begrenzung derselben wegen Mondscheins nicht genauer feststellen. - Aug. 18. Beobachtung fortwährend durch Wolken unterbrochen. - Aug. 19. Luft dunstig; Comet sehr schwer zu sehen. Zeitweilig musste die Beobachtung wegen eintretender Trübung unterbrochen werden. Später wird es klarer, doch konnte auch dann nur eine schwache Beleuchtung der Fäden angewandt werden. - Aug. 21 . Der Kern des Cometen gleicht einem Stern 1 1.-1 2. Gr. Der Coma geht eine blasse Nebelmasse voraus. - Aug. 24. Beobachtung fortwährend durch Wolken unterbrochen. Der Kern des Cometen erscheint doppelt. - Aug. 3o. Ein heller sternartiger Kern sitzt im östlichen Theile der Nebelhülle, die sich ziemlich weit nach Westen ausbreitet. - Sept. 8. Ausgezeichnete Bilder. Wegen des ausgeprägten Kerns lässt der Comet sich scharf einstellen. Während der letzten Durchgänge erschien der Comet wegen des Mondscheins und eines leichten Dunstschleiers, der diesen Theil des Himmels bedeckte, sehr schwach. - Sept. 10. Der Comet hat ein etwas verändertes Aussehen. Der an der östlichen Begrenzung der Coma befindliche Kern erscheint weniger

scharf, als bisher. Nach Westen erstreckt sich eine fächerförmig sich ausbreitende Nebelmasse. - Sept. 22. Sehr durchsichtige Luft. Der Kern erscheint hell und scharf, wodurch eine genaue Einstellung sehr begünstigt wird. Eine ausgedehnte Nebelmasse befindet sich nordwestlich vom Kern. - Sept. 24. Die Beobachtung wird durch einige den Cometen umstehende kleine Sterne erschwert. - Sept. 26. Durch Wolken beobachtet. Wegen beginnenden Regens abgebrochen. - Sept. 30. Nordwestlich vom Kern leuchtet in $3^{\prime \prime}-4^{\prime \prime}$ Entfernung bisweilen ein zweites Lichtptinktchen auf, wodurch die Pointirung auf den Kern erschwert wird. Im Laufe der Beobachtung zeigte sich, dass es an der Cometenbewegung theilnimmt. - Oct. x. Wiederum glaube ich einen zweiten Kern zu sehen in derselben Entfernung, wie am Tage vorher. - Oct. 7. Bei den Distanzmessungen mussten die äussersten Windungen der Mikrometerschraube benutzt werden. - Oct. 19. Der Comet ist merklich schwächer geworden. Er besteht aus einer ziemlich ausgebreiteten Nebelmasse, in deren östlichem Theile sich ein scharfer kleiner Kern = einem Sternchen 13. Gr. befindet. - Oct. 20. Comet schwach. - Oct. 22. Wegen der grossen Distanz kamen die äussersten Gänge der Mikrometerschraube in Anwendung. - Oct. 27. Dunst. Ungemein schwierige Beobachtung. Comet selbst auf ganz dunklem Felde nur mit Mühe zu sehen.

Die Veröffentlichung einiger weiterer Beobachtungen kann erst erfolgen, wenn mir zuverlässige Vergleichsternpositionen zu Gebote stehen werden.

Pulkowa 1893 Januar.

F. Renz.

") Die AR. der Ephemeride ist $21 \$ 50$ zu lesen statt $21 \$ 30$ (vgl. A. N. 3151 p. 1I I). 